\title{
STALing B cell responses with CD22
}

\author{
Craig P. Chappell and Edward A. Clark
}

Department of Immunology, University of Washington, Seattle, Washington, USA.

\begin{abstract}
Suppressing unwanted humoral immune responses without compromising the host's ability to respond to foreign pathogens is a primary goal for therapies aimed at ameliorating harmful autoantibody production. Global suppression of the immune system via lymphocyte depletion and/or immunosuppressive drugs can have off-target effects, a limitation to conventional therapies. In this issue of the JCI, Macauley and colleagues utilize a novel platform to inhibit antigen-specific antibody production that preserves the immune system's ability to respond to unrelated antigens.
\end{abstract}

$\mathrm{B}$ cells are critical for providing protection from disease because the Abs they produce offer an important first line of defense from invading pathogens. However, B cells can also contribute to harmful immune responses especially when their $\mathrm{Ab}$ production is directed toward self antigens (Ags), as is the case in many autoimmune diseases. Finding ways to manipulate B cell responses - either enhancing them for vaccine development or inhibiting them in patients with autoimmune and allergic diseases - has been a long-standing goal for vaccinologists and clinicians alike. One promising strategy that has emerged for immune modulation is the targeted delivery of Ag to specific immune cell subsets (1). This approach uses mAbs or ligands specific for cell surface receptors to deliver linked Ags directly to immune cells upon injection in vivo. In this issue of the JCI, Macauley and colleagues introduce a novel Ag-targeting approach to inhibit B cell responses that combines both $\mathrm{Ag}$ specificity and negative regulation of $\mathrm{B}$ cell receptor (BCR) signaling (2).

\section{Activation versus inhibition: STALing B cells}

Upon binding Ags via their BCRs, B cells become activated and differentiate into plasma cells that produce Ab. Direct inhibition of Ag-specific B cells is a difficult task to achieve because any Ag-specific therapy directed at $\mathrm{B}$ cells must, by its very nature, incorporate $\mathrm{Ag}$ binding to the $\mathrm{BCR}$ and hence risk inducing activation versus inhibition. To circumvent this obstacle, Macauley and colleagues took advantage of

Conflict of interest: Edward A. Clark has a research contract with UCB Pharmaceuticals.

Citation for this article: J Clin Invest. 2013; 123(7):2778-2780. doi:10.1172/JCI69670.
CD22, a sialic acid-binding BCR coreceptor highly expressed on the B cell surface (2). They chose CD22 because its cytoplasmic tail contains 2 immunotyrosine-inhibitory motifs that are phosphorylated upon BCR ligation and recruit the $\mathrm{SH} 2$ domaincontaining protein tyrosine phosphatase SHP-1 (3, 4). SHP-1 dephosphorylates multiple components of the BCR signaling cascade, resulting in suppression of BCR-mediated signaling. Pursuing the hypothesis that excessive crosslinking of CD22 would prohibit BCR signal transduction and therefore Ag-specific $\mathrm{Ab}$ responses, Macauley et al. generated liposomal nanoparticles containing optimized ratios of $\mathrm{Ag}$ and the high-affinity ligand

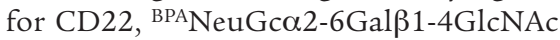
(referred to hereafter as CD22 ligand, or CD22L). Administration of these SIGLECengaging tolerance-inducing antigenic liposomes (STALs, where SIGLEC is defined as sialic acid-binding Ig-like lectin) to mice significantly decreased IgM and IgG Ab responses upon subsequent challenge with the same particles that lack CD22L (immunogenic liposomes). The induction of $\mathrm{B}$ cell tolerance by STALs was demonstrated for several different Ags including both $\mathrm{T}$ cell-independent and $\mathrm{T}$ cell-dependent Ags. Both CD22 and BCR engagement were required on the same cell to induce tolerance: liposomes containing only CD22L did not globally suppress B cell responses, and STALs did not suppress $A b$ responses to unrelated Ags. Thus, the tolerance induced by STALs was Ag-specific (2).

Macauley et al. demonstrated that tolerance induction by STALs required the presence of CD22, as no tolerance was induced in Cd22-deficient mice, and administration of immunogenic liposomes enhanced Agspecific $\mathrm{Ab}$ responses instead of inhibiting them. STAL-induced B cell tolerance was also associated with strong inhibition of BCR signaling components, consistent with CD22 recruitment of SHP-1. Somewhat unexpectedly, STAL treatment led to apoptosis of B cells in vitro and depletion of Agspecific (but not polyclonal) B cells in vivo. Although the precise mechanism of cell death was not determined, STAL treatment led to accumulation of FoxO transcription factors in the nucleus (2), which are known to regulate expression of genes that influence cell-cycle status and apoptosis (5).

The strong inhibition of $\mathrm{B}$ cell responses by STALs demonstrates the power that costimulatory/secondary signals have in determining how $\mathrm{B}$ cells respond when they recognize Ag via the BCR. Consistent with this notion, $B$ cells can also serve as efficient targets for enhancement of $\mathrm{Ab}$ responses if given the proper signals. Early efforts to enhance Ag-specific B cell responses focused on targeting the BCR for Ag delivery using antiimmunoglobulin Abs $(6,7)$. These attempts demonstrated that targeting reduces the quantity of Ags required for activated helper T cells, yet they generally failed to show robust enhancement of $\mathrm{Ab}$ responses, probably because additional signals beyond BCR ligation itself were not provided. Ig levels are elevated when mice are injected with an $\mathrm{Ab}$ to CD180, a TLR orphan receptor highly expressed on B cells with homology to TLR4 (8), and Ag coupled to anti-CD180 induces rapid Ag-specific $\mathrm{Ab}$ production in vivo (our unpublished results). Thus, depending on which receptor is targeted in vivo along with the BCR on a B cell, either Ag-specific B cell inhibition (CD22) or activation (CD180) is induced (Figure 1).

\section{Antigen targeting: DC or B cell?}

Ag targeting is an attractive approach for manipulating Ag-specific immune responses because it offers a number of advantages that conventional therapies do not. Because Ag is delivered directly to specific and often rare cell populations, it reduces the quantity of $\mathrm{Ag}$ required to initiate immune responses. Most importantly, it allows control over which cell types receive and present Ag to the immune system. This is a critical aspect for any Ag-specific therapy because APCs directly influence the 


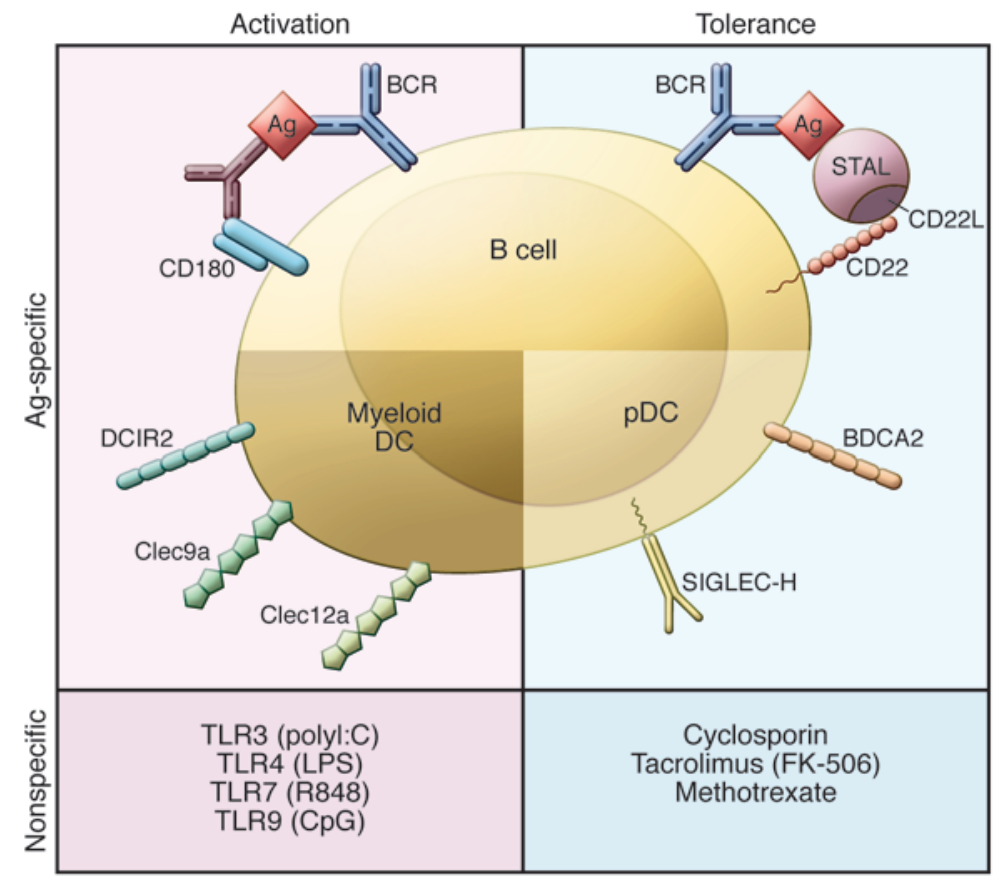

\section{Figure 1}

Multiple cellular and molecular targets for modulating Ag-specific B cell responses. Schematic of methods to achieve Ag-specific vs. nonspecific B cell activation or inhibition. STALs directly induce Ag-specific B cell tolerance by simultaneously targeting Ag to the BCR together with inhibitory signals delivered by CD22 ligands. Alternatively, delivery of Ag to the BCR together with CD180 crosslinking results in Ag-specific $B$ cell activation and Ab production. B cell responses may also be modulated by Ag delivery to receptors found on DC subsets. Ag targeted to SIGLEC-H or BDCA2 found on pDCs indirectly inhibits B cell responses via inhibition of effector CD4 ${ }^{+} \mathrm{T}$ cell activation, whereas Ag-specific $\mathrm{B}$ cell responses can be induced by delivering Ag to certain C-type lectin receptors found on myeloid DCs. B cell responses can be enhanced or inhibited by nonspecific agents such as TLR agonists or immunosuppressive drugs, respectively.

type of immune response that is induced, e.g. $\mathrm{CD}^{+}, \mathrm{CD}^{+}$, or $\mathrm{B}$ cell responses $(9,10)$. Due to their central role in initiating immunity or tolerance, most Ag-targeting studies have focused on DCs. DCs are a heterogeneous population of APCs and thus offer multiple choices as targets that can be used to modulate immune responses. For instance, Ag delivery to one of several surface receptors found on CD8 $\alpha^{-}$DCs in mice including DC inhibitory receptor 2 (DCIR2), F4/80-like immune receptor (FIRE), or C-type lectin immune receptor (CIRE) lead to induction of robust Ag-specific $A b$ responses $(11,12)$. In the case of $A g$ targeting to DCIR2, CD8 $\alpha^{-}$DCs directly induced $\mathrm{B}$ cells to differentiate into $\mathrm{Ab}$ producing plasma cells.

On the other hand, inhibition of Ag-specific $\mathrm{Ab}$ responses by DCs can be achieved through Ag delivery to SIGLEC-H (13) or blood-derived cell antigen 2 (BDCA2) found on plasmacytoid DCs (pDCs) (our unpublished results). As opposed to STALs, which directly suppress BCR signal transduction, the inhibition of $\mathrm{Ab}$ responses by $\mathrm{pDCs}$ operates via inhibition of $\mathrm{CD}^{+} \mathrm{T}$ cells, thus denying $\mathrm{B}$ cells the $\mathrm{CD}^{+} \mathrm{T}$ cell help critical for $\mathrm{T}$ cell-dependent $\mathrm{Ab}$ responses. A final advantage of Ag-targeting strategies is the ability to include immune-modulating compounds or agonists that can be chosen based on the expression of inhibitory or activating receptors expressed by the targeted cell type. DC-induced $\mathrm{Ab}$ responses can be enhanced by administering costimulatory signals, such as TLR7 or TLR9 agonists, together with the targeted $\operatorname{Ag}(11,14)$. Likewise, crosslinking BDCA2 on pDCs has been shown to negatively regulate interferon production, indicating it may have a role in promoting immune tolerance $(15,16)$. Thus, Ag delivery to DCs has the capacity to regulate $\mathrm{B}$ cell responses either directly or indirectly, depending on both the receptor that is targeted and the type of costimulation (activating or inhibitory) that is delivered along with the Ag. Figure 1 summarizes the cellular and molecular targets that have been successfully used to modulate Ag-specific B cell responses.

\section{STALing hemophilia}

Inhibiting harmful $\mathrm{Ab}$ production by $\mathrm{B}$ cells is not only desired in autoimmune settings, but has tremendous potential in cases in which anti-drug Abs are prohibitive, particularly for biologic-based therapeutics. To demonstrate the potential applicability of STALs in a clinical setting, Macauley et al. chose a mouse model of hemophilia A in which factor VIII (FVIII) deficiency leads to an inability to prevent bleeding (2). Hemophilia A affects approximately 1 in 10,000 males, who must be continuously treated with recombinant human FVIII (rhFVIII) (termed replacement therapy) to prevent uncontrolled bleeding (17). Approximately $20 \%-30 \%$ of patients receiving replacement therapy develop anti-FVIII antibodies that preclude further treatment with rhFVIII (18). Immune tolerance induction (ITI), which requires repeated administration of highdose FVIII over a period of months to years, is the current treatment for development of anti-FVIII Abs (19). Although ITI is often successful, it is extremely costly, requires frequent injections that make venous access difficult, increases risk of infection, and is less likely to work in patients with high titers of anti-FVIII Abs. Treatment of FVIII-deficient mice with FVIII-containing STALs significantly reduced the development of anti-FVIII Abs upon challenge with immunogenic liposomes. Strikingly, when these mice were administered rhFVIII replacement therapy, they were able to prevent significant blood loss in a tail-cut assay and were indistinguishable from untreated mice given one round of rhFVIII just prior to tail cutting. In contrast, mice that did not receive rhFVIII, or mice pretreated with immunogenic liposomes prior to replacement therapy, were unable to prevent significant blood loss (2).

\section{Moving forward}

The ability to inhibit anti-FVIII Abs clearly demonstrates the value STALs may provide in these settings. However, it is less clear how STAL treatment would affect ongoing B cell responses, especially in the context of autoimmune diseases where additional inflammatory "second signals" may be present that could break tolerance (Figure 1). This is 
particularly relevant to auto-Ags that consist of DNA- or RNA-containing immune complexes. Although $\mathrm{Ab}$ responses to some Ags moderately increased following STAL treatment, $100 \%$ effective depletion of Ag-specific $\mathrm{B}$ cells may not be necessary, as a reduction (vs. abolishment) in harmful auto-Abs may be sufficient for clinical benefit. Considering that most autoimmune disorders also contain a significant $\mathrm{T}$ cell component, it may be necessary to combine STAL treatment with additional therapies aimed at inhibiting $\mathrm{T}$ cell responses such as costimulatory blockade or Ag targeting to pDCs. Despite these questions, the new platform introduced by Macauley and colleagues opens an exciting avenue for $\mathrm{B}$ cell manipulation, and adds to an ever-growing body of work that seeks to manipulate Ag-specific immune responses.

\section{Acknowledgments}

We thank Christiane Dresch for critical comments. Our research described here was supported by NIH grants R01-AI52203 and R37-AAI4257 to E.A. Clark.

Address correspondence to: Edward A. Clark, Department of Immunology, Uni- versity of Washington, Office I-319A, HSC, Box 357242, 1959 NE Pacific St. NE, Seattle, Washington 98195, USA. Phone: 206.543.8706; Fax: 206.616.6772; E-mail: eaclark@uw.edu.

1. Caminschi I, Lahoud MH, Shortman K. Enhancing immune responses by targeting antigen to DC. Eur J Immunol. 2009;39(4):931-938.

2. Macauley MS, et al. Antigenic liposomes displaying CD22 ligands induce antigen-specific B cell apoptosis. J Clin Invest. 2013;123(7):3074-3083.

3. Otipoby KL, Draves KE, Clark EA. CD22 regulates $\mathrm{B}$ cell receptor-mediated signals via two domains that independently recruit Grb2 and SHP-1.J Biol Chem. 2001;276(47):44315-44322.

4. Nitschke L. CD22 and Siglec-G: B-cell inhibitory receptors with distinct functions. Immunol Rev. 2009;230(1):128-143.

5. Burgering BM, Medema RH. Decisions on life and death: FOXO Forkhead transcription factors are in command when $\mathrm{PKB} /$ Akt is off duty. J Leukoc Biol. 2003;73(6):689-701.

6. Denis O, Latinne D, Nisol F, Bazin H. Resting B cells can act as antigen presenting cells in vivo and induce antibody responses. Int Immunol. 1993;5(1):71-78.

7. Kawamura H, Berzofsky JA. Enhancement of antigenic potency in vitro and immunogenicity in vivo by coupling the antigen to anti-immunoglobulin. Immunol. 1986;136(1):58-65.

8. Chaplin JW, Kasahara S, Clark EA, Ledbetter JA. Anti-CD180 (RP105) activates B cells to rapidly produce polyclonal Ig via a T cell and MyD88-independent pathway. J Immunol. 2011;187(8):4199-4209.

9. Pulendran B, Tang H, Denning TL. Division of labor, plasticity, and crosstalk between dendritic cell subsets. Curr Opin Immunol. 2008;20(1):61-67.

10. Dudziak D, et al. Differential antigen processing by dendritic cell subsets in vivo. Science. 2007; 315(5808):107-111

11. Chappell CP, Draves KE, Giltiay NV, Clark EA. Extrafollicular B cell activation by marginal zone dendritic cells drives $\mathrm{T}$ cell-dependent antibody responses. J Exp Med. 2012;209(10):1825-1840.

12. Corbett AJ, et al. Antigen delivery via two molecules on the CD8- dendritic cell subset induces humoral immunity in the absence of conventional "danger". Eur I Immunol. 2005;35(10):2815-2825.

13. Loschko J, et al. Antigen targeting to plasmacytoid dendritic cells via Siglec-H inhibits Th cell-dependent autoimmunity. IImmunol. 2011;187(12):6346-6356.

14. Lahoud MH, et al. The C-type lectin Clec12A present on mouse and human dendritic cells can serve as a target for antigen delivery and enhancement of antibody responses. J Immunol. 2009;182(12):7587-7594.

15. Fanning SL, et al. Receptor cross-linking on human plasmacytoid dendritic cells leads to the regulation of IFN-alpha production. J Immunol. 2006; 177(9):5829-5839.

16. Rock J, et al. CD303 (BDCA-2) signals in plasmacytoid dendritic cells via a BCR-like signalosome involving Syk, Slp65 and PLCgamma2. Eur J Immunol. 2007;37(12):3564-3575

17. Ehrenforth S, et al. Incidence of development of factor VIII and factor IX inhibitors in haemophiliacs. Lancet. 1992;339(8793):594-598.

18. Stonebraker JS, Bolton-Maggs PH, Michael Soucie J, Walker I, Brooker M. A study of variations in the reported haemophilia $B$ prevalence around the world. Haemophilia. 2010;18(3):e91-e94.

19. Dimichele DM. Management of factor VIII inhibitors. Int J Hematol. 2006;83(2):119-125.

\title{
Linking MLL and the HGF-MET signaling pathway in liver cancer
}

\author{
Jens U. Marquardt'1,2 and Snorri S. Thorgeirsson'1
}

\begin{abstract}
${ }^{1}$ Laboratory of Experimental Carcinogenesis (LEC), Center for Cancer Research, National Cancer Institute, NIH, Bethesda, Maryland, USA
\end{abstract} 2Department of Medicine I, Johannes Gutenberg University, Mainz, Germany.

\begin{abstract}
Mixed-lineage leukemia (MLL; also known as myeloid/lymphoid), the human homolog of trithorax in Drosophila, is a transcriptional coactivator that plays an essential role during early development and hematopoiesis. Furthermore, MLL is critically involved in the epigenetic regulation of cell cycle, senescence, DNA damage, and stem cell self-renewal. Chromosomal aberrations of $M L L$ in acute leukemias are well documented, but the role of this gene in solid malignancies remains unclear. In this issue of the JCI, Takeda et al. describe a novel epigenetic link between MLL and the HGFMET signaling pathway conferring invasive and metastatic properties to hepatocellular carcinoma cells.
\end{abstract}

The genomic landscapes of most common forms of human cancer have now been defined (1). One of the most interesting findings emerging from these studies

Conflict of interest: The authors have declared that no conflict of interest exists.

Citation for this article: J Clin Invest. 2013;

123(7):2780-2783. doi:10.1172/JCI70235. was the identification of approximately 140 genes that, when altered by intragenic mutations, can promote or "drive" tumorigenesis in human cancers. Importantly, these driver genes can be classified into one or more of 12 signaling pathways regulating three fundamental cellular processes: cell fate, cell survival, and genome maintenance (1). It is self-evident that better understanding and therapeutic use of these signaling pathways promises to improve both treatment and prevention of human cancer.

Among the druggable driver genes are several key oncogenic molecules, such as EGFR, BRAF, and MET, motivating the development of targeted agents that have already improved the outcome of the subgroups of patients with malignancies harboring activating mutations in these genes. However, in hepatocellular carcinomas (HCCs), no such clear "oncogene addiction” exists, highlighting a role for alternative modes of oncogene activation, such as by epi-driver genes, which are epigenetically altered to be aberrantly expressed in cancer, conferring a growth advantage $(1,2)$. HCC most often develops in the context of a chronically inflamed microenviron- 